\title{
IMPLEMENTATION OF GRADIENT METHODS FOR OPTIMIZATION OF UNDERAGE COSTS IN AVIATION INDUSTRY
}

\author{
Nataša Kontrec $^{1 *}$, Milena Petrović ${ }^{1}$
}

${ }^{1}$ Faculty of Mathematics and Sciences, University of Priština, Kosovska Mitrovica, Serbia.

\begin{abstract}
Underage costs are not easily quantifiable in spare parts management. These costs occur when a spare part is required and none are available in inventory. This paper provides another approach to underage cost optimization for subassemblies and assemblies in aviation industry. The quantity of spare parts is determined by using a method for

airplane spare parts forecasting based on Rayleigh's model. Based on that, the underage cost per unit is determined by using the Newsvendor model. Then, by implementing a transformed accelerated double-step size gradient method, the underage costs for spare sub-assemblies and assemblies in airline industry are optimized.
\end{abstract}

Key words: cost optimization, gradient methods, spare parts.

\section{INTRODUCTION}

The main goal of this paper is to present an innovative implementation of gradient methods in optimization of underage costs for spare parts in aviation industry. The gradient methods represent methods for systematic research and solution finding in an iterative procedure for unconstrained optimization problems. The underage cost is defined as the cost per unit of product in case of negative inventory level at the end of the product's usability period. All airline companies share a common goal of minimizing these costs on one side and increasing the level of aircraft's availability on the other. Hence, this paper further elaborates on some of the methods of unconditional optimization.

\section{THEORETICAL PART}

Due to the fact that minimizing underage costs is critical, the problem will be presented as follows.

Minimizing given objective function is required:

$$
(\min ) \mathrm{f}(x), \quad x \in R^{n},
$$

where for some point $x^{*}$ the expression (1) is satisfied. Defined point $x^{*}$ can be described trough the next two definitions:

Def: Point $x^{*}$ is the local minimum point if there exists a neighborhood $\mathrm{U}\left(x^{*}\right)$ such that

$$
f\left(\mathrm{x}^{*}\right) \leq f(\mathrm{x})
$$

for each vector $x \in U\left(x^{*}\right)$.
Point $x^{*}$ is the point of strict local minimum if there exists a neighborhood $U\left(x^{*}\right)$ such that

$$
f\left(\mathrm{x}^{*}\right)<f(\mathrm{x})
$$

for each vector $x \neq x^{*}$ and $x \in U\left(x^{*}\right)$.

Def: Point $x^{*}$ is the global minimum point if

$$
f\left(\mathrm{x}^{*}\right) \leq f(\mathrm{x}), \quad \forall \mathrm{x} \in \mathrm{R}^{n} .
$$

Then, the neighborhood $U\left(x^{*}\right)$ of that point exists for each vector $x \neq x^{*}$ and $x \in U\left(x^{*}\right)$ such that:

$$
f\left(\mathrm{x}^{*}\right)<f(\mathrm{x}), \quad \forall \mathrm{x} \in \mathrm{R}^{n} .
$$

The practice proved it was easier to find local minimum points than finding the global minimum point. Thus, finding the global minimum point could be reduced to determination of several local minimums and then identifying the smallest. The smallest one will be declared as the global minimum. Finding the local minimum is even easier if the search is conducted at the descending vector direction. If the objective function is differentiable, than the gradient method should be used.

As stated above, the gradient methods are numerical iterative methods. In order to determine the minimal function value by using such a method, it is necessary to define the size of the iterative step and search direction since both are crucial elements of each gradient iterative scheme. There are several iterative methods, each defined in a specific way, relevant for this work. Some of them are presented in articles (Andrei, 2006), (Stanimirović et al., 2010), 
(Petrović et al., 2014), (Petrović et al., 2015), (Stanimirović et. al. 2015). After their comparison, it has been concluded that the TADSS method (Stanimirović et. al., 2015) (Transformed Accelerated Double Step Size) outperforms all other gradient iterative methods.

The essence of the TADSS method is in proper setting of numerical iterations, in order to obtain a satisfying approximate solution to the problem. The iteration process for this method is defined as:

$$
x_{k+1}=x_{k}-\left[\alpha_{k}\left(\gamma_{k}^{-1}-1\right)+1\right] g_{k},
$$

where $\alpha_{k}$ denotes the size of the iterative step - can be computed as in algorithm 1. Since the TADSS is an accelerated optimization method, the acceleration parameter that presents a multiplying factor of the size of the iterative step has to be defined. Determination of this factor is based on Taylor's expansion and appropriated approximation of Hessian and can be calculated as:

$$
\gamma_{k+1}=2 \frac{f\left(x_{k}+1\right)-f\left(x_{k}\right)+\left(\alpha_{k}\left(\gamma_{k}^{-1}-1\right)+1\right)\left\|g_{k}\right\|^{2}}{\left(\alpha_{k}\left(\gamma_{k}^{-1}-1\right)+1\right)^{2}\left\|g_{k}\right\|^{2}}
$$

where $g_{k}$ denotes a gradient vector of objective function at the $k$-th iterative point.The iterative step, according to this method, can be computed as in (7). The size of the iterative step $\alpha_{k}$ can be determined by using the following backtracking procedure:

Algorithm 8: Backtracking line search procedure for calculating iterative step $\alpha_{\mathrm{K}}$ starting at $\alpha=1$

Requirements: Objective function $f(x)$, the direction $d_{k}$ of the search at the point $x_{k}$, real numbers $0<\sigma<0.5$ and $\eta \in(0, \sigma)$

1. Set $\alpha=1$.

2. While $f\left(x_{k}+\alpha d_{k}\right)>f\left(x_{k}\right)+\sigma \alpha g_{k} d_{k}$, take $\alpha:=\eta \alpha$.

3. Return $\alpha_{\mathrm{K}}=\alpha$.

Algorithm 9: Transformed accelerated double step size method (TADSS method)

Requirements: $0<\rho<1,0<\tau<1, x_{0}, \gamma_{0}=1$.

1. Set $k=0$, compute $f\left(x_{0}\right), g_{0}$ and take $\gamma_{0}=1$.

2. If $\left\|g_{k}\right\|<\varepsilon$ then go to Step 8, else go to the next step.

3. Find the step $\alpha_{\kappa}$ applying the Algorithm 1.

4. Compute the next interative point $x_{k+1}$ using (6).

5. Determine scalar $\gamma_{k+1}$ using (7)

6. If $\gamma_{k+1}<0$ take $\gamma_{k+1}=1$.

7. Set $k:=k+1$, and go to the Step 2 .

8. Return $x_{k+1}$ and $f\left(x_{k+1}\right)$.

\section{EXPERIMENTAL}

\subsection{Method evaluation and presentation}

An innovative model for spare parts assessment in aviation industry is presented in paper (Kontrec et al., 2015). This paper also includes a method for determining the required quantities of spare parts in inventory and, on those basis, determining the underage costs per unit of product. As these costs refer to individual parts, the goal of this paper is to optimize the underage costs for subassemblies or assemblies comprised of several such parts.

If the quantity of spare parts is determined, as conducted in paper (Kontrec et al., 2015), then the underage costs can be calculated by using the Newsvendor model (Hill, 2011). The Newsvendor model is widely known model used mostly when evaluation of a certain stochastic variable is required. This evaluation is a compromise between the loss that has occurred when the value of stochastic variable is underestimated and the loss that will occur if the value of that stochastic variable is overestimated, as follows:

$$
q=\Phi^{-1}\left(\frac{c_{u}}{c_{o}+c_{u}}\right)
$$

$\Phi^{-1}$ denotes Inverse distribution function; $c_{o}$ denotes cost per unit of product in case of positive level of inventory at the end of the product's usability period; $c_{u}$ denotes the underage cost i.e. cost of unmet demand for spare part.

As on the basis of formula (10) only underage costs for individual parts can be determined, in cases where entire assembly or subassembly need to be replaced, minimizing underage costs can be conducted by using the TADSS method.

In that case, the objective function can be expressed as in (11):

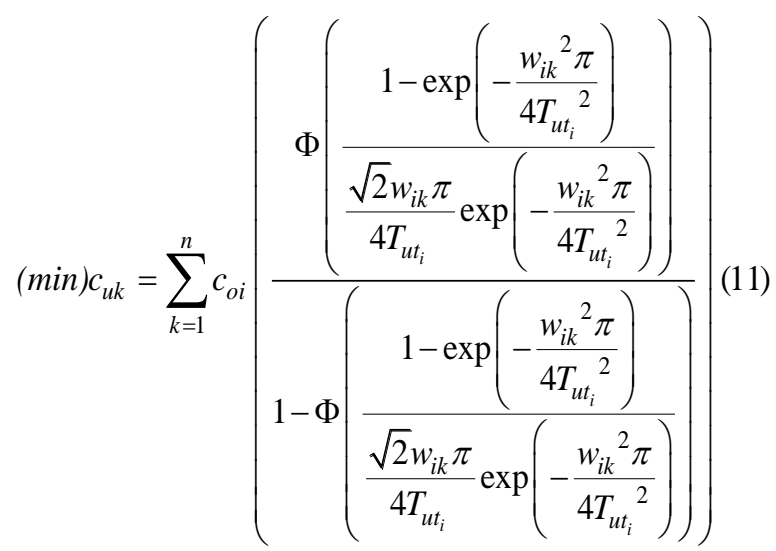

$i=1, \ldots, m$ 
Wherein:

$c_{u k}$ - underage cost in certain flight hour $w_{k}, k=1, \ldots, n$ $c_{o i}$-price per unit of the part of the assembly, where $i=1, \ldots, m$, andmrepresents the number of parts comprised in the assembly.

$T_{u t i}$-total unit time of $i$-th part comprised in the assembly.

Decision variables are:

$$
w_{k}=\left(w_{1 k}, w_{2 k}, \ldots, w_{n k}\right),
$$

Initial values of decision variables are values from environment of points that represent the life cycle duration of each part:

$$
w_{0}=\left(T_{u t_{1}}-\Delta T_{u t_{1}}, T_{u t_{2}}-\Delta T_{u t_{2}}, \ldots, T_{u t_{n}}-\Delta T_{u t_{n}}\right)
$$

If we implement TADSS method to the defined objective function (10), we can conclude that the size of the iterative step is:

$$
w_{k+1}=w_{k}-\left[\alpha_{k}\left(\gamma_{k}^{-1}-1\right)+1\right] g_{k},
$$

while the acceleration parameter can be expressed as follows:

$$
\gamma_{k+1}=2 \frac{f\left(w_{k}+1\right)-f\left(w_{k}\right)+\left(\alpha_{k}\left(\gamma_{k}^{-1}-1\right)+1\right)\left\|g_{k}\right\|^{2}}{\left(\alpha_{k}\left(\gamma_{k}^{-1}-1\right)+1\right)^{2}\left\|g_{k}\right\|^{2}}
$$

Based on all of the aforementioned, the objective function gradient can be calculated as stated in (16):

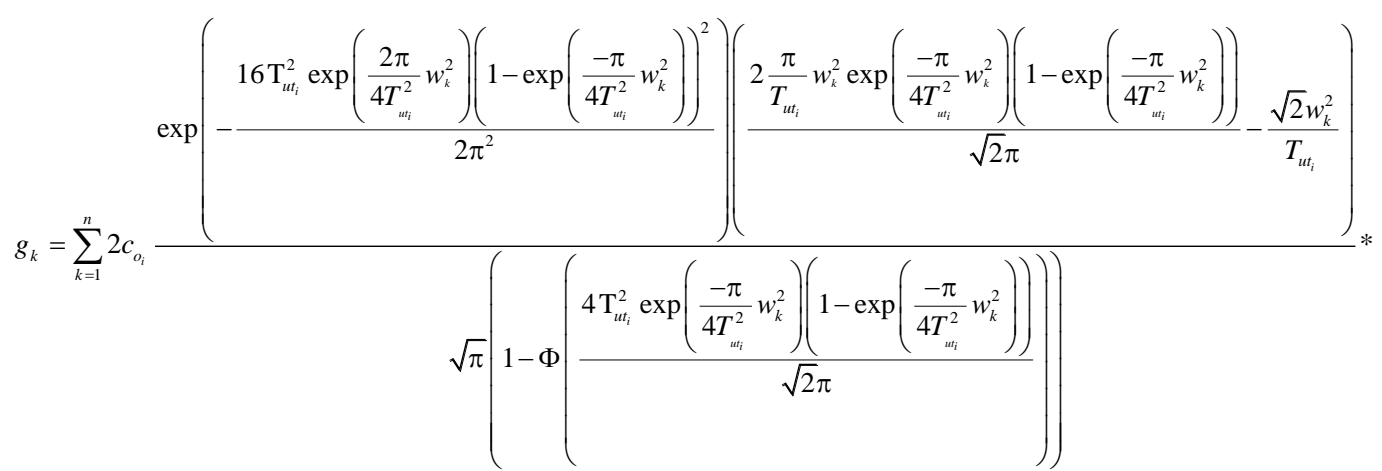

$$
\begin{aligned}
& *\left(\frac{\left(\frac{4 \mathrm{~T}_{u t_{i}}^{2} \exp \left(\frac{-\pi}{4 T_{w_{i}}^{2}} w_{k}^{2}\right)\left(1-\exp \left(\frac{-\pi}{4 T_{w_{i}}^{2}} w_{k}^{2}\right)\right)}{\sqrt{2} \pi}\right)}{\left(1-\Phi\left(\frac{4 \mathrm{~T}_{u_{i}}^{2} \exp \left(\frac{-\pi}{4 T_{u_{i}}^{2}} w_{k}^{2}\right)\left(1-\exp \left(\frac{-\pi}{4 T_{u_{i}}^{2}} w_{k}^{2}\right)\right)}{\sqrt{2} \pi}\right)\right)}+1\right)
\end{aligned}
$$

When the total unit time $\mathrm{T}_{\mathrm{ut}}$ of assemblies/subassemblies' constitutive parts and prices of those parts are known, then on the basis of (16) their minimum underage costs in specific time intervals can be determined. These intervals or inspection points can be determined as conducted in papers (Wu, 2013), (Tian, 2013).

\section{RESULTS AND DISCUSSION}

Main contribution of this paper is to provide the possibility to minimize underage costs for spare assemblies and subassemblies in aviation industry. As previously stressed, these costs are hard to quantify. In most cases, only a rough assessment of these costs is conducted. These assessments are complex and hardly ever completely objective and impartial. Some consequences of the lack of spare parts, such as damage to a company's reputation due to unforeseen delays, are almost impossible to quantify as cost. Hence, the contribution of this paper is even more significant. Moreover, the gradient methods have not been utilized in cost optimization until now. This paper combines the Transformed Accelerated Double Step Size method, method for spare parts inventory forecasting based on Rayleigh's model and the Newsvendor model. If the total unit time for each part of the assembly or subassembly and prices of those parts are known, it is possible to determine the 
underage cost in preselected time intervals, based on the proposed method.

\section{REFERENCES}

Andrei, N. 2006. An acceleration of gradient descent algorithm with backtracking for unconstrained optimization. Numerical Algorithms, 42(1), pp. 6373.

Hill, A. V. 2011. The Newsvendor Problem. Clamshell Beach Press.

Kontrec, N., Milovanović, G.V., Panić, S., \& Milošević, H. 2015. A Reliability-Based Approach to Nonrepairable Spare Part Forecasting in Aircraft Maintenance System. Mathematical Problems in Engineering, 2015. Article ID731437, 7 pages, doi:10.1155/2015/731437.

Petrović, M. J., \& Stanimirović, P.S. 2014. Accelerated double direction solving unconstrained optimization problems. Mathematical Problems in Engineering, . Article ID965104, 8 pages.

\footnotetext{
*E-mail: natasa.kontrec@pr.ac.rs
}

Petrović, M. J. 2015. An accelerated double step size model in unconstrained optimization. Applied Mathematics and Computation, 250, pp. 309-319.

Stanimirović, P. S., \& Miladinović, M.B. 2010. Accelerated gradient descent methods with line search. Numerical Algorithms, 54(4), pp. 503-520.

Stanimirović, P. S., Milovanović, G.V., Petrović, M.J., \& Kontrec, N. 2015. A Transformation of Accelerated Double Step Size Method for Unconstrained Optimization. Mathematical Problems in Engineering, 2015(8). Article ID2836798 pages. doi:10.1155/2015/283679.

Tian, Z., Wu, B., \& Chen, M. 2013. Condition based maintenance optimization considering inproving prediction accuracy. Journal of the Operational Research Society, 65(9), pp. 1412-1422.

Wu, B., Tian, Z., \& Chen, M. 2013. Condition-based maintenance optimization using neural networkbased health condition prediction. Quality and Reliability Engineering International, 29(8), pp. 1151-1163. 